\title{
Orthorhombic distortion and orbital order in the vanadium spinel $\mathrm{FeV}_{2} \mathrm{O}_{4}$
}

\author{
S. Kawaguchi, ${ }^{1,2}$ H. Ishibashi, ${ }^{2}$ S. Nishihara, ${ }^{3}$ S. Mori, ${ }^{4}$ J. Campo,${ }^{5}$ F. Porcher,${ }^{6}$ O. Fabelo, ${ }^{7}$ K. Sugimoto, ${ }^{1}$ J. Kim, ${ }^{1}$ K. Kato, ${ }^{8}$ \\ M. Takata, ${ }^{8}$ H. Nakao, ${ }^{9}$ and Y. Kubota ${ }^{2}$ \\ ${ }^{1}$ Japan Synchrotron Radiation Research Institute, SPring-8, Sayo, Hyogo 679-5198, Japan \\ ${ }^{2}$ Department of Physical Science, Graduate School of Science, Osaka Prefecture University, Sakai, Osaka 599-8531, Japan \\ ${ }^{3}$ Department of Chemistry, Hiroshima University, 1-3-1 Kagamiyama, Higashi-Hiroshima 739-8530, Japan \\ ${ }^{4}$ Department of Material Science, Graduate School of Engineering, Osaka Prefecture University, Sakai, Osaka 599-8531, Japan \\ ${ }^{5}$ Instituto de Ciencia Materiales de Aragón, CSIC-Universidad de Zaragoza, 50009 Zaragoza, Spain \\ ${ }^{6}$ Laboratoire Léon Brillouin, CEA/Saclay, Gif-sur-Yvette 91191, France \\ ${ }^{7}$ Institut Laue Langevin, 71 avenue des Martyrs, Grenoble 38042, France \\ ${ }^{8}$ RIKEN SPring-8 Center, Sayo, Hyogo 679-5148, Japan \\ ${ }^{9}$ Condensed Matter Research Center and Photon Factory, IMSS, KEK, Tsukuba, Ibaraki 305-0801, Japan
}

(Received 30 April 2015; revised manuscript received 4 November 2015; published 13 January 2016)

\begin{abstract}
Using synchrotron and neutron diffraction measurements, we find a low-temperature orthorhombic phase in vanadium spinel $\mathrm{FeV}_{2} \mathrm{O}_{4}$. The orbital order of $\mathrm{V}^{3+}$ ions with tetragonal normal modes occurs at $68 \mathrm{~K}$, and this leads to an appearance of the pseudotetragonal phase at a noncollinear ferrimagnetic transition temperature. Below the magnetic transition temperature, unconventional behavior of the orbital state of $\mathrm{Fe}^{2+}$ ions accompanied by the emergence of the orthorhombic phase was observed by using the normal mode analysis. We have also studied the structural properties of orbitally diluted materials. The orthorhombic phase, which is significantly affected by the other ions, is intrinsic in $\mathrm{FeV}_{2} \mathrm{O}_{4}$. We suggest the orthorhombic phase is strongly related with the double orbital states of $\mathrm{Fe}^{2+}$ and $\mathrm{V}^{3+}$ ions.
\end{abstract}

DOI: 10.1103/PhysRevB.93.024108

\section{INTRODUCTION}

Obtaining an accurate crystal structure is critically important for understanding the interplay of orbital and spin degrees of freedom and lattice distortions, because it provides significant clues to the understanding of the behavior of its lattice degrees of freedom [1,2]. Recently, vanadium spinel oxides $A \mathrm{~V}_{2} \mathrm{O}_{4}(A=\mathrm{Mn}, \mathrm{Co}, \mathrm{Fe}, \mathrm{Zn}$, and $\mathrm{Cd})$, which have the $\mathrm{V}^{3+}\left(3 d^{2}\right)$ ions in triply degenerate $t_{2 \mathrm{~g}}$ levels at octahedral sites ( $B$ sites), have been intensively investigated as one of the forefront topics in modern orbital physics.

Most of these compounds exhibit a cubic to compressed tetragonal $(c<a)$ lattice distortion caused by the orbital order $(\mathrm{OO})$ of $\mathrm{V}^{3+}$ ions at low temperature, the origins of which have been experimentally and theoretically studied [3-11]. However, $\mathrm{FeV}_{2} \mathrm{O}_{4}$ is an unconventional material because an elongated tetragonal phase $(c>a)$ appears at low temperature, and the $A$ sites occupied by $\mathrm{Fe}^{2+}$ ions have orbital degrees of freedom in the $e$ states [12]. Hence, $\mathrm{FeV}_{2} \mathrm{O}_{4}$ has Jahn-Teller (JT) active ions at both the $A$ sites and the $B$ sites; thus, the competition of ions, the cooperation of ions, or both induce multistep structural and magnetic transitions $[11,12]$ accompanied by unique physical properties, i.e., multiferroic nature, large magnetostriction, and anomalous magnetization [12-17]. Therefore, a detailed structural study of $\mathrm{FeV}_{2} \mathrm{O}_{4}$ is important for understanding the origin of physical properties and the contribution to orbital physics. However, the OO models of $\mathrm{V}^{3+}$ ions at $T_{\mathrm{N} 2}$, suggested by Nii et al. [11] and Kang et al. [18], are contradictory. Additionally, the origins and structural properties of the low-temperature phase including the elongated tetragonal phases [12] are still disputed despite numerous experimental and theoretical efforts [11-21]. In this paper, we tackle the accurate structure analysis with high-resolution synchrotron diffraction and neutron diffraction by using polycrystalline samples as well as single crystals of $\mathrm{FeV}_{2} \mathrm{O}_{4}$ and orbitally diluted materials for each $A$ and $B$ site.

\section{EXPERIMENTAL}

The powder samples of $\mathrm{FeV}_{2} \mathrm{O}_{4}, \mathrm{Fe}_{1-x} \mathrm{Mn}_{x} \mathrm{~V}_{2} \mathrm{O}_{4}$, and $\mathrm{FeV}_{2-x} \mathrm{Fe}_{x} \mathrm{O}_{4}$ used in this study were prepared by solid-state reaction from a stoichiometric mixture of high-purity $\mathrm{Fe}_{2} \mathrm{O}_{3}$, $\mathrm{Fe}, \mathrm{V}_{2} \mathrm{O}_{3}$, and $\mathrm{MnO}$ powder. The powder was heated in an evacuated quartz tube at $1323 \mathrm{~K}$ for $24 \mathrm{~h}$ in a furnace, and the resultant sample was subsequently ground and pressed into pellets under $35 \mathrm{MPa}$ pressure. The pellet was sintered in an evacuated tube at $1373 \mathrm{~K}$ for $24 \mathrm{~h}$. The single crystal was grown using a floating-zone method (see Appendix A). The compositions of these samples were measured by energy dispersive $\mathrm{x}$-ray spectroscopy. The compositions in powder samples almost correspond to those for preparation; however, excess $\mathrm{Fe}$ of $5 \%-10 \%$ exists in the single crystal, which is consistent with the past studies [12,17]. In this paper, we express the single crystal of $\mathrm{FeV}_{2} \mathrm{O}_{4}$ as $\mathrm{Fe}_{1+\delta} \mathrm{V}_{2-\delta} \mathrm{O}_{4}$ single crystal (see Appendix A).

Synchrotron powder diffraction (SPD) experiments were carried out with a large Debye-Scherrer camera installed at the beamline BL02B2 at SPring-8 [22]. The wavelength of the incident $x$ ray was $0.79790 \AA$. The sample temperature was controlled by the $\mathrm{He} / \mathrm{N}_{2}$ gas flow devices. The singlecrystalline synchrotron diffraction experiment was performed at BL-4C with an off-center-type four-circle diffractometer (Photon Factory, KEK). The x-ray beam was monochromatized to $10.33 \mathrm{keV}(1.2 \AA)$. The single crystal $(2.5 \times 2.0 \times$ $1.5 \mathrm{~mm}^{3}$ ) was mounted with the $[100]_{\mathrm{c}}$ axis parallel to the scattering vector $\boldsymbol{q}$ in the closed-type cryostat. The neutron powder diffraction (NPD) measurements were carried out at high-flux (G41) and high-resolution powder diffractometers 
(3T2) at the Laboratoire Léon Brillouin and the Institut Laue-Langevin. The wavelengths of incident beams at G41 and 3T2 were 2.42834 and $1.22494 \AA$, respectively. The magnetization and specific heat measurements were performed by the SQUID magnetometer (MPMS-XL) and the physical property measurement system, respectively.

\section{SYNCHROTRON AND NEUTRON DIFFRACTION MEASUREMENTS}

Figure 1(a) shows the synchrotron powder diffraction (SPD) and neutron powder diffraction (NPD) patterns around the 440 cubic Bragg reflections at low temperatures. In the SPD patterns, the distinguishing feature is that three peaks are observed at $15 \mathrm{~K}$, indicating an orthorhombic phase. In contrast, the peak splitting at other temperatures is similar to that in past studies [12]. In the NPD study, signs of these transitions were also observed, consistent with recent literature $[13,20,21]$. However, the peak splitting was not observed as clearly as in the SPD study because of the lower angular resolution of NPD. Therefore, we mainly focused on SPD experiments. We performed structural refinements by the Rietveld method and obtained the crystallographic parameters listed in Tables I and II. Figures 1(b) and 1(c) give the results of the Rietveld fitting in the SPD pattern at $15 \mathrm{~K}$ and the NPD pattern at $2 \mathrm{~K}$, respectively. These refinements are best described by the orthorhombic model with the space group $F d d d$. This space group is a subgroup of the tetragonal space group $I 4_{1} / a m d$, and it has been often applied to describe structural ground states of the spinels with JT active ions located at the $A$ site [23]. The characteristic atomic displacement (mentioned in Sec. IV) was found in the low-temperature orthorhombic (LTO) phase, whereas the spin alignments and moment sizes for the $\mathrm{Fe}^{2+}$ and $\mathrm{V}^{3+}$ cations [Fig. 1(d)] were almost the same as those in the canted-ferrimagnetic structure reported in the literature [13].

The temperature dependencies of magnetization and specific heat are shown in Fig. 1(e), and those of the lattice constants, which are calculated from SPD data, are shown in Fig. 1(f). These results indicate structural transitions from cubic to high-temperature tetragonal (HTT; $c<a$ ) at $T_{\mathrm{S} 1}=137 \mathrm{~K}$, HTT to high-temperature orthorhombic (HTO) at $T_{\mathrm{N} 1}=106 \mathrm{~K}$, HTO to low-temperature tetragonal (LTT; $c>a$ ) at $T_{\mathrm{N} 2}=68 \mathrm{~K}$, and LTT to LTO at $T_{\mathrm{S} 2} \approx 30 \mathrm{~K}$. Note that the crystal axes in the HTT and LTT phases are defined as $\vec{a}_{\mathrm{HTT}}=(1 / 2)(\vec{a}-\vec{b}), \vec{b}_{\mathrm{HTT}}=(1 / 2)(\vec{a}+\vec{b}), \vec{c}_{\mathrm{HTT}}=\vec{c}, \vec{a}_{\mathrm{LTT}}=$ $(-1 / 2)(\vec{c}+\vec{b}), \vec{b}_{\mathrm{LTT}}=(-1 / 2)(\vec{c}-\vec{b})$, and $\vec{c}_{\mathrm{LTT}}=\vec{a}$, where $\vec{a}$, $\vec{b}$, and $\vec{c}$ are the cubic lattice vectors, and the lengths of $\vec{a}, \vec{b}$, and $\vec{c}$ are shown in Fig. 1(f) as $a, b$, and $c$, respectively. Considering previous neutron diffraction experiments [13], $T_{\mathrm{N} 1}$ and $T_{\mathrm{N} 2}$ represent collinear ferrimagnetic (C-FM) and noncollinear ferrimagnetic (NC-FM) orders, respectively, accompanied by lattice distortions. The magnetic structures refined using our NPD data agree well with those in the literature [13]. However, an anomaly accompanied by the emergence of the LTO phase was not observed in the temperature dependencies of magnetization and specific heat; the reason for this will become evident later. We also performed single-crystalline synchrotron diffraction; however, an indication of lattice distortion from LTT to LTO below $30 \mathrm{~K}$ was not clearly detected (see
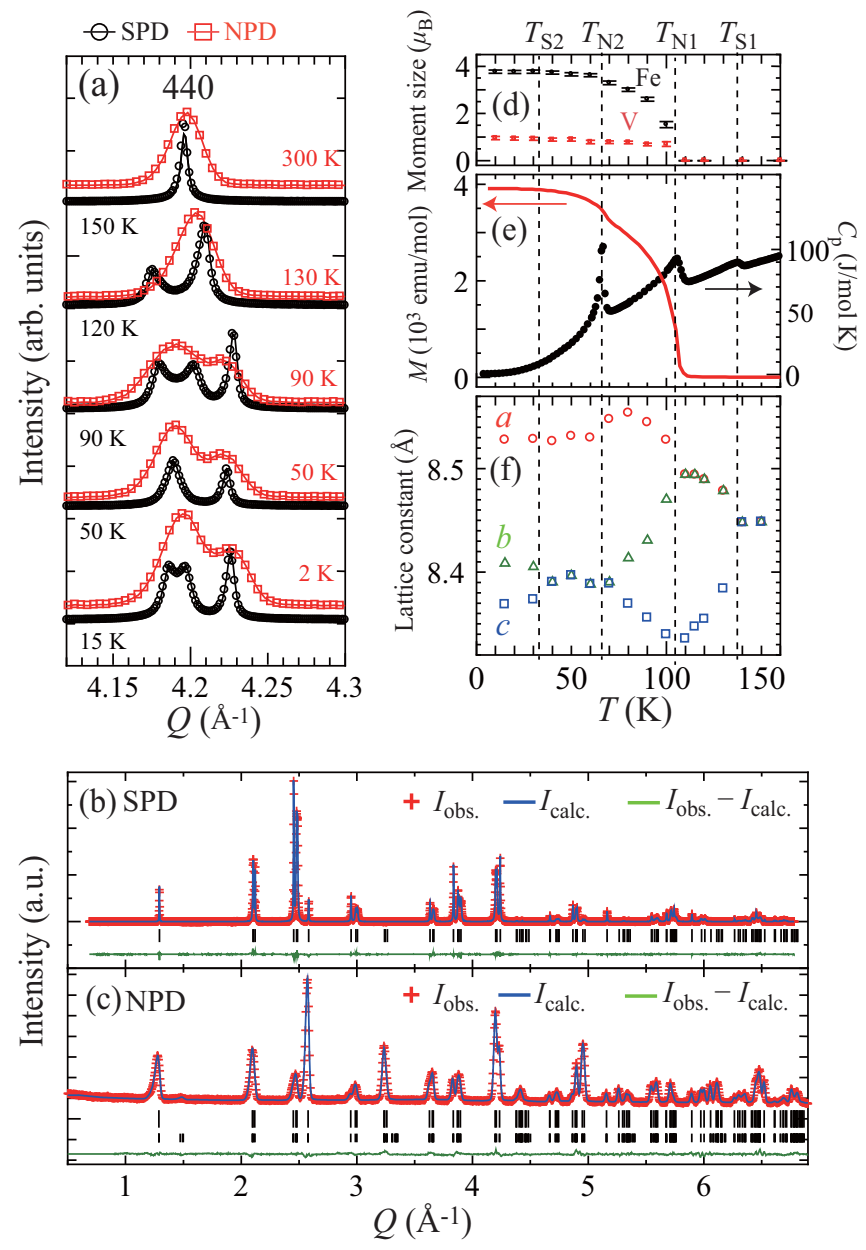

FIG. 1. Temperature dependence of the (a) SPD and NPD patterns around 440 cubic Bragg reflection. Rietveld fitting for (b) SPD pattern at $15 \mathrm{~K}$ and (c) NPD pattern at $2 \mathrm{~K}_{\text {in }} \mathrm{FeV}_{2} \mathrm{O}_{4}$. The crosses, solid lines, and bottom lines represent the observed intensities, the calculated ones, and the differences, respectively. The vertical bars show the position of Bragg peaks (for the NPD pattern, the long and short bars indicate the crystal and magnetic reflections, respectively) with the $F d d d$ space group. The temperature dependencies of (d) the ordered moment size on the $\mathrm{Fe}^{2+}$ and $\mathrm{V}^{3+}$ cation sites, (e) the magnetization (left axis) as well as the heat capacity (right axis), and (f) the lattice constants in $\mathrm{FeV}_{2} \mathrm{O}_{4}$. The lattice constant in the temperature range of $T_{\mathrm{S} 2} \leqslant T \leqslant T_{\mathrm{N} 2}$ was calculated by using the LTT model with the $I 4_{1} /$ amd space group. Note that the lattice constants in the HTT and LTT phases are shown by the cubic axes for comparison. Typical errors of the lattice constants are less than the size of the data markers. Note that the moments below $T_{\mathrm{N} 1}$ are calculated using the $F d d d$ model.

Appendix B) presumably because of excess $\mathrm{Fe}$ in the single crystal due to evaporation of $\mathrm{Fe}$ and separation of $\mathrm{V}_{2} \mathrm{O}_{3}$ in the synthesis (see Appendix A).

\section{ORBITAL STATES OF Fe ${ }^{2+}$ AND V ${ }^{3+}$ IONS}

To understand the origin of structural phase transitions, we investigated the detailed orbital states of both $\mathrm{Fe}^{2+}$ and $\mathrm{V}^{3+}$ ions in the LTO phase as well as other phases, and we performed normal $(Q)$ mode analysis, which is related 
TABLE I. Structural parameters in $\mathrm{FeV}_{2} \mathrm{O}_{4}$. The cubic and orthorhombic phases have the space groups $F d \overline{3} m$ and $F d d d$ (origin choice 1), respectively, and then the tetragonal phases have the space group $I 4_{1} /$ amd (origin choice 2 ).

\begin{tabular}{|c|c|c|c|c|c|c|c|}
\hline & Cubic & Cubic & HTT & HTO & LTT & LTT & LTO \\
\hline$T$ & $300 \mathrm{~K}$ & $150 \mathrm{~K}$ & $115 \mathrm{~K}$ & $100 \mathrm{~K}$ & $60 \mathrm{~K}$ & $40 \mathrm{~K}$ & $15 \mathrm{~K}$ \\
\hline$a(\AA)$ & $8.45361(5)$ & $8.44846(7)$ & $6.0062(2)$ & $8.5278(2)$ & $5.93142(6)$ & $5.93289(8)$ & $8.5277(1)$ \\
\hline$b(\AA)$ & 8.45361 & 8.44846 & 6.0062 & $8.4703(2)$ & 5.93142 & 5.93289 & 8.4091(1) \\
\hline$c(\AA)$ & 8.45361 & 8.44846 & $8.3472(2)$ & $8.3398(2)$ & $8.5299(1)$ & $8.5264(1)$ & $8.3687(1)$ \\
\hline$Z$ & 8 & 8 & 4 & 8 & 4 & 4 & 8 \\
\hline $\mathrm{Fe}^{\mathrm{a}}$ & $8 a$ & $8 a$ & $4 a$ & $8 a$ & $4 a$ & $4 a$ & $8 a$ \\
\hline$B\left(\AA^{2}\right)$ & $0.75(1)$ & $0.46(1)$ & $0.46(2)$ & $0.44(2)$ & $0.39(2)$ & $0.33(4)$ & $0.27(2)$ \\
\hline $\mathrm{V}^{\mathrm{b}}$ & $16 d$ & $16 d$ & $8 d$ & $16 d$ & $8 d$ & $8 d$ & $16 d$ \\
\hline$B\left(\AA^{2}\right)$ & $0.56(1)$ & $0.36(1)$ & $0.50(2)$ & $0.46(2)$ & $0.38(1)$ & $0.22(3)$ & $0.32(2)$ \\
\hline $\mathrm{O}$ & $32 e$ & $32 e$ & $16 h$ & $32 h$ & $16 h$ & $16 h$ & $32 h$ \\
\hline$x$ & $0.3867(1)$ & $0.3867(1)$ & 0 & $0.3879(4)$ & 0 & 0 & $0.3910(2)$ \\
\hline$y$ & 0.3867 & 0.3867 & $0.4747(4)$ & $0.3862(4)$ & $0.4827(2)$ & $0.4843(6)$ & $0.3842(3)$ \\
\hline$z$ & 0.3867 & 0.3867 & $0.2584(3)$ & $0.3823(3)$ & $0.2662(1)$ & $0.2656(4)$ & $0.3831(2)$ \\
\hline$B\left(\AA^{2}\right)$ & $0.67(3)$ & $0.59(3)$ & $0.28(3)$ & $0.31(3)$ & $0.18(2)$ & $0.10(6)$ & $0.11(3)$ \\
\hline$R_{\mathrm{WP}}(\%)$ & 4.75 & 5.50 & 4.81 & 5.03 & 2.44 & 6.54 & 3.09 \\
\hline$R_{\mathrm{I}}(\%)$ & 3.31 & 2.89 & 3.59 & 3.60 & 2.33 & 2.41 & 2.59 \\
\hline
\end{tabular}

${ }^{a}$ Fractional coordinates $(x, y, z)$ in the $8 a$ and $4 a$ sites are $(0,0,0)$ and $(0,3 / 4,1 / 8)$, respectively.

${ }^{\mathrm{b}}$ Fractional coordinates $(x, y, z)$ in the $16 d$ and $8 d$ sites are $(5 / 8,5 / 8,5 / 8)$ and $(0,0,1 / 2)$, respectively.

to the local distortions of the ligands [11,24-28]. Figure 2 represents the distortions of the $\mathrm{FeO}_{4}$ tetrahedron and the $\mathrm{VO}_{6}$ octahedron in the two-dimensional $Q_{2}-Q_{3}$ plane, where $Q_{2}$ and $Q_{3}$ correspond to orthorhombic and tetragonal distortions, respectively.

First, we focus on the distortion of the $\mathrm{FeO}_{4}$ tetrahedron, considering the $Q_{2}$ and $Q_{3}$ modes related to the $e$ orbital states. A negative $Q_{3}\left(\theta=\pi\right.$ related to the $3 z^{2}-r^{2}$ type orbital) mode was observed at $115 \mathrm{~K}$, and a positive $Q_{2}$ mode appeared at $100 \mathrm{~K}$. Subsequently, at $60 \mathrm{~K}$, the $y^{2}-z^{2}$ orbitals are stabilized where $\theta=2 \pi / 3$. These behaviors are explained by the cooperative JT effect at $T_{\mathrm{S} 1}$ and the relativistic spin-orbit (SO) coupling at $T_{\mathrm{N} 1}$ of the $\mathrm{Fe}^{2+}$ ions, which stabilize the $3 z^{2}-r^{2}$ and $y^{2}-z^{2}$ orbitals [11,25,27], respectively. Here, we introduce the deviation angle $\Delta \theta$ from $\theta=\pi$ (Fig. 2), which indicates the variation of orbital states between $3 z^{2}-r^{2}$ and $y^{2}-z^{2}$ orbitals. The most distinguishing feature is the variation of $\Delta \theta$ from $60^{\circ}$ at $60 \mathrm{~K}$ to $52^{\circ}$ at $15 \mathrm{~K}$. This unconventional behavior, which was not seen in other materials with orbital degrees of freedom, is likely related to the two orbitally active ions.

Next, the distortions of the $\mathrm{VO}_{6}$ octahedron are discussed in the $Q_{2}-Q_{3}$ plane. The $\mathrm{VO}_{6}$ octahedron was compressed along the $x$ direction below $T_{\mathrm{N} 2}$, similar to the behavior observed in the $\mathrm{Fe}_{1+\delta} \mathrm{V}_{2-\delta} \mathrm{O}_{4}$ single crystal [11]. This distortion is very similar to that derived from the ferro-OO type where one electron occupies the $y z$ orbital and other occupies the $(x y+i z x) / \sqrt{2}$ orbital [11]. We found that the $Q_{2}$ and $Q_{3}$ values of the $\mathrm{VO}_{6}$ octahedron at $15 \mathrm{~K}$ are almost equal to those at $60 \mathrm{~K}$.

\section{ORTHORHOMBIC DISTORTION}

To investigate the nature of the orthorhombic lattice at the ground state and the variation of $\Delta \theta$ below $T_{\mathrm{N} 2}$, we examined the SPD profiles of $\mathrm{FeV}_{2} \mathrm{O}_{4}$ in detail. The crosses in Fig. 3(a)

TABLE II. O-Fe-O bond angle, $\mathrm{V}-\mathrm{O}$ bond distance, and $\mathrm{O}-\mathrm{V}-\mathrm{O}$ bond angle in $\mathrm{FeV}_{2} \mathrm{O}_{4}$.

\begin{tabular}{|c|c|c|c|c|c|c|c|}
\hline & Cubic & Cubic & HTT & HTO & LTT & LTT & LTO \\
\hline$T$ & $300 \mathrm{~K}$ & $150 \mathrm{~K}$ & $115 \mathrm{~K}$ & $100 \mathrm{~K}$ & $60 \mathrm{~K}$ & $40 \mathrm{~K}$ & $15 \mathrm{~K}$ \\
\hline \multirow[t]{3}{*}{$\mathrm{O}-\mathrm{Fe}-\mathrm{O}$ angle (deg) } & $109.47(7)$ & $109.47(7)$ & $103.43(9)$ & $107.3(1)$ & $105.56(7)$ & $105.5(1)$ & $105.65(9)$ \\
\hline & & & $108.1(1)$ & $108.9(1)$ & $111.46(6)$ & $111.5(1)$ & $110.91(9)$ \\
\hline & & & & $112.4(1)$ & & & $111.93(9)$ \\
\hline \multirow[t]{3}{*}{ V-O distance $(\AA)$} & $2.0189(9)$ & $2.0181(9)$ & $2.020(2)$ & $2.025(4)$ & $1.997(1)$ & $2.001(4)$ & $1.998(2)$ \\
\hline & & & $2.022(3)$ & $2.027(4)$ & $2.0307(8)$ & $2.037(3)$ & $2.031(2)$ \\
\hline & & & & $2.029(4)$ & & & $2.031(2)$ \\
\hline \multirow[t]{6}{*}{$\mathrm{O}-\mathrm{V}-\mathrm{O}$ angle (deg) } & $84.22(7)$ & $84.25(7)$ & $83.83(9)$ & $84.1(1)$ & $83.96(7)$ & $84.3(1)$ & $83.86(9)$ \\
\hline & $95.78(7)$ & $95.75(7)$ & $84.8(1)$ & $85.1(1)$ & $85.66(6)$ & 86.1(1) & $84.12(9)$ \\
\hline & & & $95.2(1)$ & $85.4(1)$ & $94.34(6)$ & $93.9(1)$ & $85.65(9)$ \\
\hline & & & $96.17(9)$ & $94.6(1)$ & $96.04(7)$ & $95.7(1)$ & $94.35(9)$ \\
\hline & & & & $95.0(1)$ & & & $95.88(9)$ \\
\hline & & & & $95.8(1)$ & & & $96.14(9)$ \\
\hline
\end{tabular}




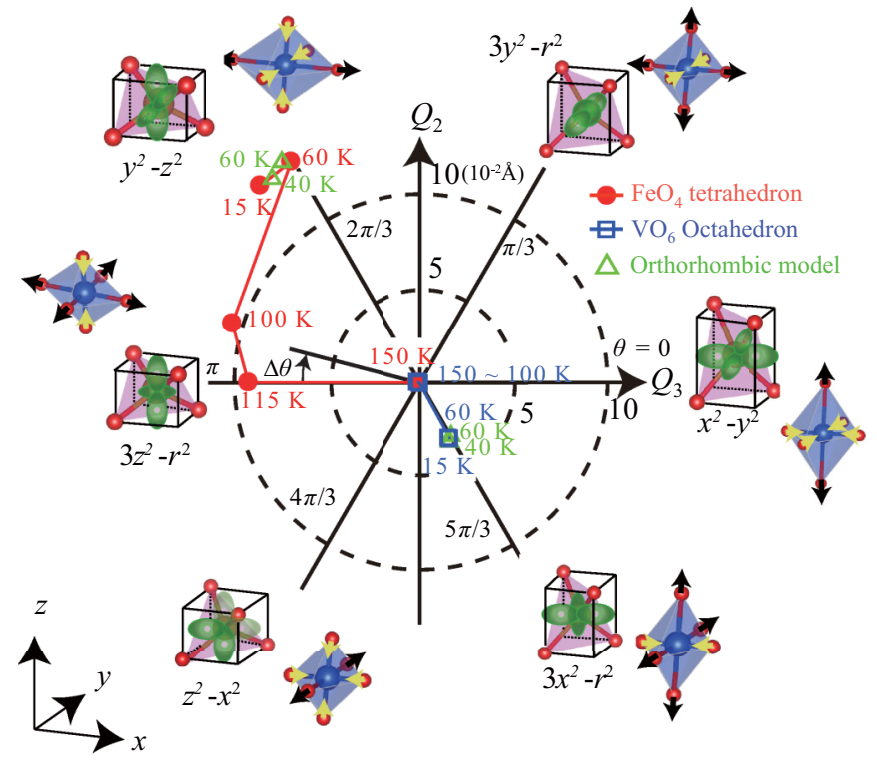

FIG. 2. Normal mode analysis in the $Q_{2}-Q_{3}$ plane of the $\mathrm{FeO}_{4}$ tetrahedron (solid circles) and $\mathrm{VO}_{6}$ octahedron (squares) in the powder sample of $\mathrm{FeV}_{2} \mathrm{O}_{4}$. The triangles represent the $Q_{2}$ and $Q_{3}$ modes of the orthorhombic model for the P-tetra phase at $T_{\mathrm{S} 2}<T<$ $T_{\mathrm{N} 2}$. Note that the $Q_{2}$ and $Q_{3}$ values, which are calculated from the SPD data, are only shown for clarity.

show the profile at $40 \mathrm{~K}$ around the 440 cubic Bragg peak. The $440_{c}$ reflection is significantly broader than that of $044_{c}$, where the subscript " $c$ " indicates the Miller indices based on the cubic axis. Additionally, the temperature dependence of the ratio of the full width at half maximum (FWHM) for the $440_{\mathrm{c}}$ to $044_{\mathrm{c}}$ reflections is shown in Fig. 3(b). The FWHM of $440_{\mathrm{c}}$ is the same as that of $044_{\mathrm{c}}$ at $T_{\mathrm{N} 1}<T<T_{\mathrm{S} 1}$, whereas the FWHM ratio below $T_{\mathrm{N} 1}$ (even in the LTT phase) is significantly larger than that in the HTT phase. This result indicates that the crystal structure in the LTT phase has orthorhombic symmetry with similar lattice constants for $b$ and $c(b \approx c)$. Furthermore, all Bragg reflections were expressed by Rietveld fitting, using the Fddd model, as shown in Figs. 3(a) and 3(c). The fitting is fairly good, and the detailed structural parameters are listed in Table III. Here, we regard the LTT phase as a pseudotetragonal (P-tetra) system with an orthorhombic lattice. The temperature dependencies of the lattice constants considering the P-tetra phase are shown in Fig. 3(d).

Structural refinements and normal mode analysis were also performed by using the orthorhombic model for the P-tetra (LTT) phase (Fig. 2; see triangles). We found only a slight change (from $\Delta \theta=60^{\circ}$ to $58^{\circ}$ ) for the $\mathrm{FeO}_{4}$ tetrahedron at $68 \mathrm{~K}$, as reflected by the orthorhombic structural models. However, the uniaxial distortion of the $\mathrm{VO}_{6}$ octahedron, which is associated with tetragonal normal modes and induced by the $\mathrm{OO}$ of $\mathrm{V}^{3+}$ ions, was not significantly influenced by the models. Here, we suggest that the behavior of $\Delta \theta$ up to near $60^{\circ}$ is caused by the ferro-OO of $\mathrm{V}^{3+}$ ions accompanied by the $\mathrm{NC}-\mathrm{FM}$ order. The $\mathrm{OO}$ of $\mathrm{V}^{3+}$ ions likely affects the orbital state of the $\mathrm{Fe}^{2+}$ ion and leads to stabilization of the $y^{2}-z^{2}$ orbital because it is associated with tetragonal normal modes at $T_{\mathrm{N} 2}$. For the variation of the lattice, the decreasing of the $a$
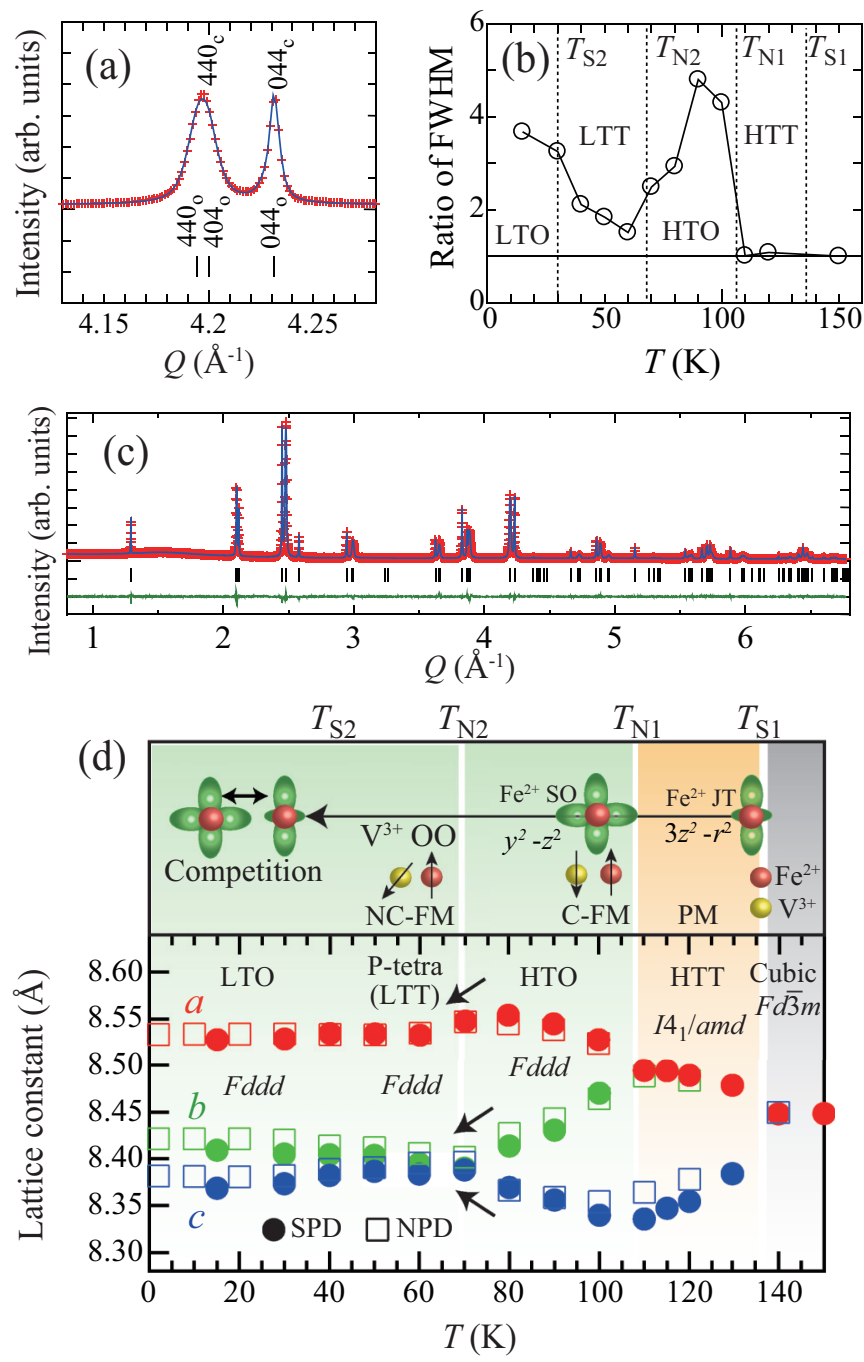

FIG. 3. (a) Rietveld fitting by using the orthorhombic model around the 440 cubic Bragg peak for SPD patterns in $\mathrm{FeV}_{2} \mathrm{O}_{4}$ at $40 \mathrm{~K}$. The subscripts "c" and "o" indicate the cubic and orthorhombic axes, respectively. (b) Temperature dependence of the ratio of the FWHM for $440_{c}$ to that for $044_{c}$ reflections. In the orthorhombic phases, the FWHM of the $440_{\mathrm{c}}$ reflection is expressed by overlapping the $440_{c}$ and $404_{c}$ reflections. (c) Rietveld analysis of $\mathrm{FeV}_{2} \mathrm{O}_{4}$ for the SPD pattern in the P-tetra phase at $40 \mathrm{~K}$. The profiled-weighted $R$ factor $R_{\mathrm{WP}}$ and the $R$-Bragg factor $R_{\mathrm{I}}$ represent $4.45 \%$ and $2.55 \%$, respectively. (d) Temperature dependencies of lattice constants for $\mathrm{FeV}_{2} \mathrm{O}_{4}$ considering the orthorhombic lattice in the P-tetra phase. Note that the lattice constant in the temperature range of $T_{\mathrm{S} 2} \leqslant T$ $\leqslant T_{\mathrm{N} 2}$ was calculated by using P-tetra model with the $F d d d$ space group. The orbital states of $\mathrm{Fe}^{2+}$ ions and the spin alignments of $\mathrm{Fe}^{2+}$ and $\mathrm{V}^{3+}$ ions are also illustrated schematically.

and $b$ axes and the increasing of the $c$ axis [see arrows in Fig. 3(d)] around $T_{\mathrm{N} 2}$ with decreasing temperature are most likely influenced by the $\mathrm{OO}$ of $\mathrm{V}^{3+}$ ions with the tetragonal normal mode of the $\mathrm{VO}_{6}$ octahedron at $T_{\mathrm{N} 2}$. Below $T_{\mathrm{N} 2}$, the orbital state of $\mathrm{Fe}^{2+}$ ions (see orbital states at $60 \mathrm{~K}$ and $15 \mathrm{~K}$ in Fig. 2) gradually changes with decreasing temperature, and then the orthorhombic structure is stabilized at the ground state. These results indicate that the lattice constants continuously change below $T_{\mathrm{N} 2}$ [Fig. 3(d)]. This continuous change of the lattice for 
TABLE III. Structural parameters of $\mathrm{FeV}_{2} \mathrm{O}_{4}$ for the P-tetra phase, where structural refinements are performed using the orthorhombic model with the space group $F d d d$ (origin choice 1).

\begin{tabular}{lcc}
\hline \hline & P-tetra $60(\mathrm{~K})$ & P-tetra $40(\mathrm{~K})$ \\
\hline$a(\AA)$ & $8.53240(8)$ & $8.5344(2)$ \\
$b(\AA)$ & $8.39482(9)$ & $8.4045(2)$ \\
$c(\AA)$ & $8.3838(1)$ & $8.3823(2)$ \\
$\mathrm{Fe}$ & $8 a(0,0,0)$ & $8 a(0,0,0)$ \\
$B\left(\AA^{2}\right)$ & $0.38(2)$ & $0.28(2)$ \\
$\mathrm{V}$ & $16 d(5 / 8,5 / 8,5 / 8)$ & $16 d(5 / 8,5 / 8,5 / 8)$ \\
$B\left(\AA^{2}\right)$ & $0.28(2)$ & $0.26(2)$ \\
$\mathrm{O}$ & $32 h$ & $32 h$ \\
$x$ & $0.3912(2)$ & $0.3911(2)$ \\
$y$ & $0.3836(6)$ & $0.3841(6)$ \\
$z$ & $0.3834(6)$ & $0.3833(6)$ \\
$B\left(\AA^{2}\right)$ & $0.44(4)$ & $0.25(4)$ \\
\hline \hline
\end{tabular}

the temperature is consistent with the results of magnetization and specific heat measurements, which show no anomalies at $T_{\mathrm{S} 2}$ in Fig. 1(e). The summary of the phase transitions and orbital states of both $\mathrm{Fe}^{2+}$ and $\mathrm{V}^{3+}$ ions are also schematically illustrated in Fig. 3(d).

\section{ORBITALLY DILUTED MATERIALS}

To ensure our suggestion that the crystal system of $\mathrm{FeV}_{2} \mathrm{O}_{4}$ at the ground state has the orthorhombic lattice, we consider the SPD profiles in the substituted materials $\left(\mathrm{Fe}_{1-x} \mathrm{Mn}_{x}\right)\left[\mathrm{V}_{2}\right] \mathrm{O}_{4}$ and $(\mathrm{Fe})\left[\mathrm{V}_{2-x} \mathrm{Fe}_{x}\right] \mathrm{O}_{4}$ (Here, parentheses and square brackets indicate the $A$ - and $B$ sites, respectively, in the spinel structure.). The detailed physical properties of these compounds are provided in Refs. [29-32]. Figure 4(a) represents the SPD patterns around the 440 cubic Bragg peaks at $15 \mathrm{~K}$ for $\left(\mathrm{Fe}_{1-x} \mathrm{Mn}_{x}\right)\left[\mathrm{V}_{2}\right] \mathrm{O}_{4}$. As $x$ increases, the $440_{\text {c }}$ reflection becomes sharper than that for $x=0.05$. The compositional dependencies of the FWHM ratio of the $440_{\mathrm{c}}$ to $044_{c}$ reflections are shown in Fig. 4(b). The ratio decreases as $x$ increases and is significantly different from unity when $x \leqslant 0.2$, whereas the FWHM for the $440_{\text {c }}$ reflection is almost equal to that for the $044_{\mathrm{c}}$ reflection when $x>0.2$. Note that the orthorhombic and compressed tetragonal phases are absent when $x \geqslant 0.3$ in these materials [29-32]. From these results, the crystal system is logically regarded as the P-tetra rather than the elongated tetragonal $(c>a)$ lattice until $x=0.2$.

We examined the distortions of the $\mathrm{FeO}_{4}$ tetrahedron and the $\mathrm{VO}_{6}$ octahedron of $\mathrm{Fe}\left[\mathrm{V}_{1.9} \mathrm{Fe}_{0.1}\right] \mathrm{O}_{4}$ in the $Q_{2}-Q_{3}$ plane. Note that the structural details are provided in a previous study [29]. Figure 5 gives the distortion of the $\mathrm{FeO}_{4}$ tetrahedron and the $\mathrm{VO}_{6}$ octahedron in the $Q_{2}-Q_{3}$ plane for polycrystalline samples of $\mathrm{FeV}_{2} \mathrm{O}_{4}$ and $\mathrm{Fe}\left[\mathrm{V}_{1.9} \mathrm{Fe}_{0.1}\right] \mathrm{O}_{4}$ and the $\mathrm{Fe}_{1+\delta} \mathrm{V}_{2-\delta} \mathrm{O}_{4}$ single crystal. Note that the structural parameters of the single crystal are found in Ref. [29]. The distortion of the $\mathrm{FeO}_{4}$ tetrahedron of polycrystalline samples of $\mathrm{FeV}_{2} \mathrm{O}_{4}$ below $T_{\mathrm{N} 2}$ is significantly different from that of the single crystal, and the unprecedented behavior ( $\Delta \theta$ from $60^{\circ}$ to $52^{\circ}$ ) disappears in the single crystal. The most distinguished feature is that
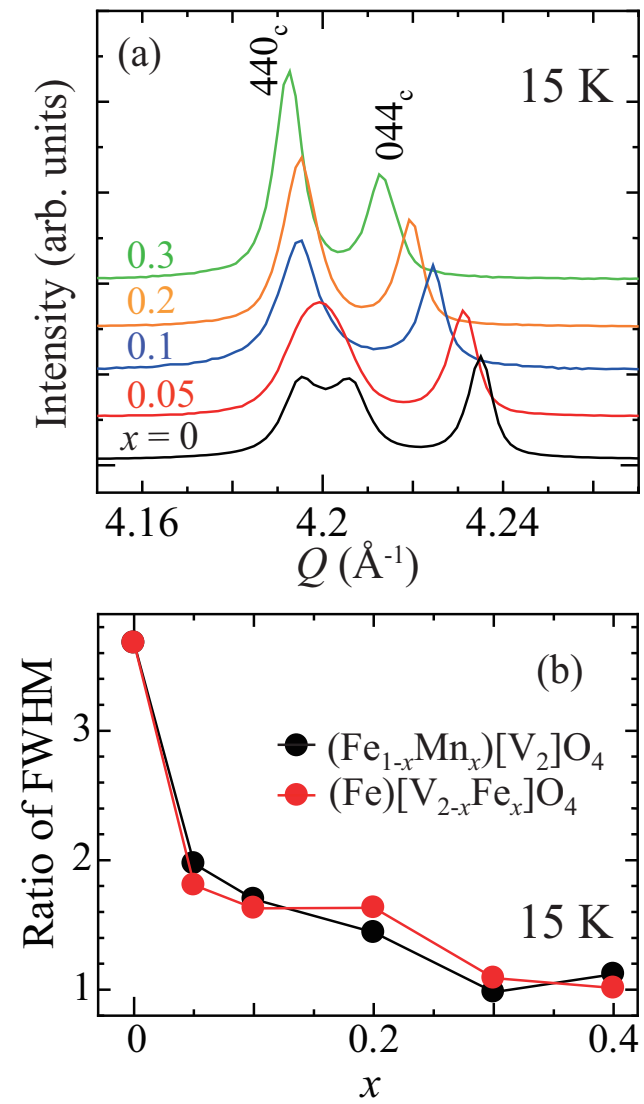

FIG. 4. (a) SPD patterns around the 440 cubic Bragg peak for $\mathrm{Fe}_{1-x} \mathrm{Mn}_{x} \mathrm{~V}_{2} \mathrm{O}_{4}$ at $15 \mathrm{~K}$. (b) Ratios of FWHM for the $440_{\mathrm{c}}$ reflection to that for the $044_{c}$ reflection in $\mathrm{Fe}_{1-x} \mathrm{Mn}_{x} \mathrm{~V}_{2} \mathrm{O}_{4}$ and $\mathrm{Fe}\left[\mathrm{V}_{2-x} \mathrm{Fe}_{x}\right] \mathrm{O}_{4}$.

the behavior of the $Q_{2}$ and $Q_{3}$ modes in $\mathrm{Fe}\left[\mathrm{V}_{1.9} \mathrm{Fe}_{0.1}\right] \mathrm{O}_{4}$ is very similar to that of the $\mathrm{Fe}_{1+\delta} \mathrm{V}_{2-\delta} \mathrm{O}_{4}$ single crystal. This is explained by excess $\mathrm{Fe}$ in $\mathrm{FeV}_{2} \mathrm{O}_{4}$, which is often observed in the single crystal and occupies the $B$ site until $x=0.35[12,17,29,33,34]$. Additionally, the orbital degrees of freedom of $t_{2 g}$ states at the $\mathrm{V}^{3+}$ site are diluted by the excess $\mathrm{Fe}$. The excess $\mathrm{Fe}$ in $\mathrm{FeV}_{2} \mathrm{O}_{4}$ makes the magnetic phase transition temperature $T_{\mathrm{N} 2}$ decrease. When the excess Fe of $10 \%$ is occupied in $\mathrm{FeV}_{2} \mathrm{O}_{4}, T_{\mathrm{N} 2}$ becomes $61 \mathrm{~K}$ [29]. This temperature well agrees with that in our $\mathrm{Fe}_{1+\delta} \mathrm{V}_{2-\delta} \mathrm{O}_{4}$ single crystal and a reported one $[13,17]$. Therefore, it is conceivable that the excess Fe always exists in the single crystal, and crystal growth of the single crystal with stoichiometry is very difficult because of the evaporation of $\mathrm{Fe}$ and the separation of $\mathrm{V}_{2} \mathrm{O}_{3}$ (see Appendix A).

On the other hand, we investigated the behavior of the $Q_{2}$ and $Q_{3}$ modes in $\mathrm{Fe}_{1-x} \mathrm{Mn}_{x} \mathrm{~V}_{2} \mathrm{O}_{4}$. The aim of this investigation is opposed to that in $(\mathrm{Fe})\left[\mathrm{V}_{2-x} \mathrm{Fe}_{x}\right] \mathrm{O}_{4}$ because the $e$ states at the $\mathrm{Fe}^{2+}$ site are diluted by the $\mathrm{Mn}^{2+}$ ion with nonorbital orbital degrees of freedom. Figure 5 shows the distortion of the $\mathrm{FeO}_{4}$ tetrahedron in the $Q_{2}-Q_{3}$ plane for polycrystalline samples of $\mathrm{Fe}_{0.9} \mathrm{Mn}_{0.1} \mathrm{~V}_{2} \mathrm{O}_{4}$. Note that the details of the structural parameters are listed in the literature [31]. Below $T_{\mathrm{N} 2}$, the behavior of $\Delta \theta$ from $60^{\circ}$ at $60 \mathrm{~K}$ to $52^{\circ}$ was not shown, and this result is similar to results in $\mathrm{Fe}\left[\mathrm{V}_{1.9} \mathrm{Fe}_{0.1}\right] \mathrm{O}_{4}$ and the $\mathrm{Fe}_{1+\delta} \mathrm{V}_{2-\delta} \mathrm{O}_{4}$ single crystal. Here, we focus on the magnitude 


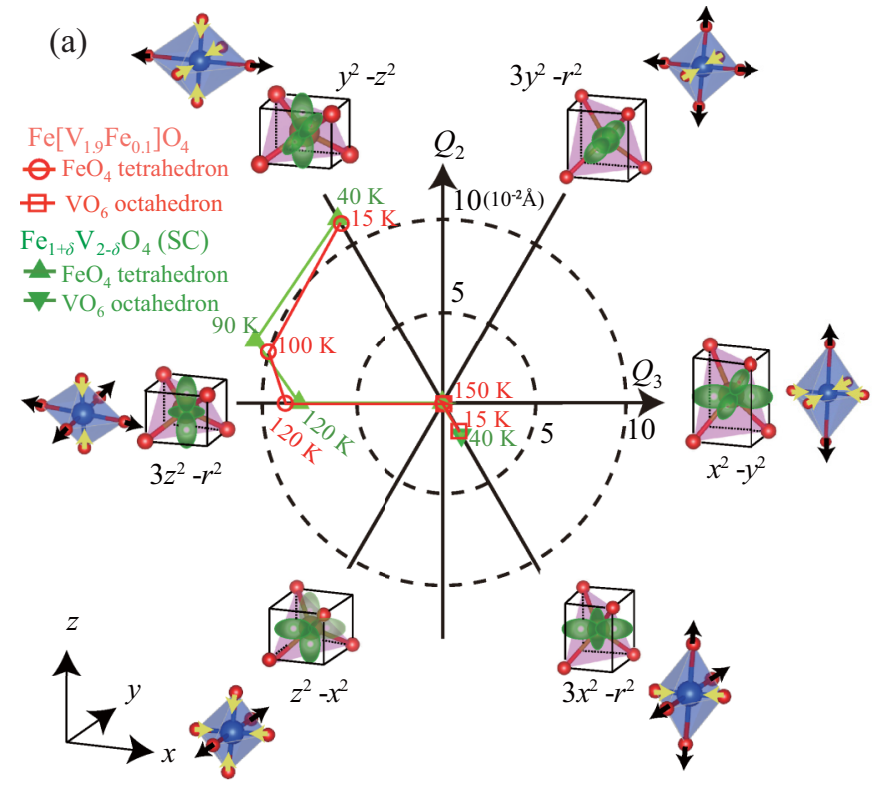

(b)

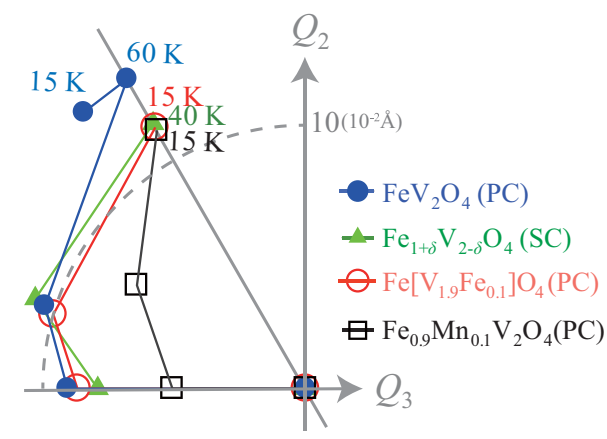

FIG. 5. (a) Normal mode analysis in the $Q_{2}-Q_{3}$ plane of the $\mathrm{FeO}_{4}$ tetrahedron and the $\mathrm{VO}_{6}$ octahedron for $\mathrm{Fe}\left[\mathrm{V}_{1.9} \mathrm{Fe}_{0.1}\right] \mathrm{O}_{4}$ and the $\mathrm{Fe}_{1+\delta} \mathrm{V}_{2-\delta} \mathrm{O}_{4}$ single crystal. Note that the $Q_{2}$ and $Q_{3}$ values in the single crystal are from Ref [11]. (b) $Q_{2}$ and $Q_{3}$ modes of the $\mathrm{FeO}_{4}$ tetrahedron for $\mathrm{FeV}_{2} \mathrm{O}_{4}, \mathrm{Fe}\left[\mathrm{V}_{1.9} \mathrm{Fe}_{0.1}\right] \mathrm{O}_{4}$, and $\mathrm{Fe}_{0.9} \mathrm{Mn}_{0.1} \mathrm{~V}_{2} \mathrm{O}_{4}$. $\mathrm{PC}$ and $\mathrm{SC}$ denote the polycrystalline and the single-crystal samples, respectively.

of distortions of the $\mathrm{FeO}_{4}$ tetrahedron and the $\mathrm{VO}_{6}$ octahedron at $15 \mathrm{~K}$ in $\mathrm{Fe}_{1-x} \mathrm{Mn}_{x} \mathrm{~V}_{2} \mathrm{O}_{4}$ [31]. The magnitudes of distortions of not only the $\mathrm{FeO}_{4}$ tetrahedron but also the $\mathrm{VO}_{6}$ octahedron are changed by the quantity of the substitution of the $\mathrm{Mn}^{2+}$ ion. This result indicates that the orbital state of the $\mathrm{Fe}^{2+}$ ion is affected by that of the $\mathrm{V}^{3+}$ ions. In these viewpoints, we suggest that not only $\mathrm{OO}$ of the $\mathrm{Fe}^{2+}$ ions but also that of the $\mathrm{V}^{3+}$ ions plays an important role in the appearance of the LTO phase. The other possible factors for suppression of the LTO phase by the substitution are the change in ionic sizes and that in valence states. In the vanadium spinel oxides, it is well known that the bond length between vanadium ions $\mathrm{V}-\mathrm{V}$ is strongly related with the localized-itinerant electronic transition, e.g., enhancement of the ferrimagnetic transition and suppression of the structural distortion [35-37]. In this study, when the ionic sizes of $\mathrm{Fe}^{2+}$ and $\mathrm{V}^{3+}$ ions are changed by the substitution of the $\mathrm{Mn}^{2+}$ and $\mathrm{Fe}^{3+}$ ions, respectively, the $\mathrm{V}-\mathrm{V}$ lengths also change. These variations can affect the electronic states in $\mathrm{FeV}_{2} \mathrm{O}_{4}$. On the other hand, the effect of the change in the valence state may be much smaller because the ionic valences at both the $A$ site and the $B$ site do not change from those of $\mathrm{FeV}_{2} \mathrm{O}_{4}$ by the small amount of the $\mathrm{Mn}^{2+}$ and $\mathrm{Fe}^{3+}$ substitution (i.e., those of the $A$ site and the $B$ site are $2+$ and $3+$, respectively) [31,34]. Therefore, the appearance of the LTO phase was possibly suppressed by the effects of not only the orbital dilution but also the change in the ionic sizes by the chemical doping. Thus, the orthorhombic lattice distortion accompanied by unprecedented behavior of orbital states of the $\mathrm{Fe}^{2+}$ ions at the lowest temperatures is suppressed by an impurity, and we conclude that the orthorhombic system at $15 \mathrm{~K}$ is an intrinsic phase in $\mathrm{FeV}_{2} \mathrm{O}_{4}$.

Meanwhile, a trigonal distortion of the $\mathrm{VO}_{6}$ octahedron has been suggested to play an important role in the $\mathrm{OO}$ of the $\mathrm{V}^{3+}$ ions and the NC-FM order at $T_{\mathrm{N} 2}$ [20]. Therefore, the appearance of the P-tetra lattice accompanied by the $\mathrm{OO}$ of $\mathrm{V}^{3+}$ ions may be related to the trigonal distortion. However, we did not observe such a distortion at $T_{\mathrm{N} 2}$, consistent with a previous study [11]. Our crystallographic studies experimentally and/or theoretically call for a reinvestigation of the physical properties in $\mathrm{FeV}_{2} \mathrm{O}_{4}$ because the orbital state of $\mathrm{V}^{3+}$ ions and the origin of the magnetostructural transition are considered only in tetragonal models $[4,5,9,10]$. Taking into account the orthorhombic structure, a detailed calculation of electronic states, local structure analysis by using highresolution transmission electron spectroscopy, and extended $\mathrm{x}$-ray absorption fine structure analysis and high-resolution neutron diffraction experiments will contribute to a further understanding of the orbital physics in vanadium spinels.

\section{CONCLUSIONS}

We found the LTO phase below $30 \mathrm{~K}$ in the polycrystalline sample of $\mathrm{FeV}_{2} \mathrm{O}_{4}$. The LTO phase was intrinsic in $\mathrm{FeV}_{2} \mathrm{O}_{4}$ because the formation of this phase was significantly affected by the impurity. We performed the structural refinements for SPD and NPD patterns using the Fddd model, and we investigated the orbital states of the $\mathrm{Fe}^{2+}$ and $\mathrm{V}^{3+}$ ions using the normal mode analysis. The unprecedented behavior of orbital states of $\mathrm{Fe}^{2+}$ ions below $T_{N 2}$ was observed. The LTO phase accompanied by this behavior disappears when the $\mathrm{Fe}^{2+}$ site and the $\mathrm{V}^{3+}$ site are occupied by the excess $\mathrm{Fe}$ and $\mathrm{Mn}^{2+}$ ions with nonorbital degrees of freedom, respectively. Hence, the formation of the LTO phase is related with double orbital states of $\mathrm{Fe}^{2+}$ ions and $\mathrm{V}^{3+}$ ions. These double degenerate OOs of $\mathrm{Fe}^{2+}$ and $\mathrm{V}^{3+}$ ions induce the unconventional structural properties below $T_{\mathrm{N} 1}$ accompanied by continuous variation of the orbital state of $\mathrm{Fe}^{2+}$ ions.

\section{ACKNOWLEDGMENTS}

This study was supported by the Ministry of Education, Culture, Sports, Science, and Technology of Japan through a Grant-in Aid for Scientific Research and by the Sasagawa Scientific Research Grant from The Japan Science Society. The synchrotron radiation experiments were performed at BL02B2 of SPring-8 with the approval of the Japan Synchrotron Radiation Research Institute (JASRI) (Proposals No. 2012A0084, No. 2012B0084, No. 2013A0084, No. 2013B0084, No. 2014A1645, and No. 2014B2049) and at BL-4C with the approval of the Photon Factory Program Committee (Proposal 
No. 2013P007). The Laboratoire Léon Brillouin and Institut Laue-Langevin are acknowledged for the allocation of neutron beam time on 3T2 and G41 (Proposals No. 10750 and No. 10748). The authors thank Mr. M. Takemoto, Dr. C. Song, and Dr. Y. Yamasaki for their help in the synchrotron diffraction experiment.

\section{APPENDIX A: SINGLE CRYSTAL GROWTH}

The crystal growth of $\mathrm{FeV}_{2} \mathrm{O}_{4}$ was carried out in an argon atmosphere in an infrared-heated image furnace installed with four halogen lamps having a power of $1500 \mathrm{~W}$ and four ellipsoidal mirrors. Figure 6 shows the quartz tube and samples in the floating-zone (FZ) system before and after crystal growth. After the growth, the black powder attaches to the inner surface of the quartz tube. This is derived from the evaporation from the rods during the crystal growth. Kismarahardja et al. [15] claimed that the evaporated powder is $\mathrm{V}_{2} \mathrm{O}_{3}$ with the corundum structure, and extra $\mathrm{V}_{2} \mathrm{O}_{3}$ in the starting materials is critical to obtain high-quality samples. However, the x-ray diffraction pattern for the black powder indicates the body-centered cubic structure with a lattice constant of $2.86 \AA$, as shown in Fig. 7(a). Additionally, we confirmed using a ferrite magnet that the black powder represents ferromagnetism at room temperature. Considering the chemical species of $\mathrm{FeV}_{2} \mathrm{O}_{4}$, we concluded that the black powder is not $\mathrm{V}_{2} \mathrm{O}_{3}$ but rather the single phase of pure $\mathrm{Fe}$.

Next, the inset of Fig. 7(a) represents the section of the seed rods after the crystal growth. The crystallinity in the inner region of growth crystal is better than that in the outer region. Accordingly, we performed the x-ray diffraction experiments using the growth crystal, which is ground into a fine powder, as shown in Fig. 7(a). In the outer of the seed rod, the peaks derived from the impurity of $\mathrm{V}_{2} \mathrm{O}_{3}$ are observed. This impurity may be caused by the evaporation of Fe. On the other hand, the inner of the crystal shows a single phase of the spinel structure. Therefore, we removed the outer region of the seed rod, and the inner of crystal was only used for this study. However, the chemical composition analysis indicates the excess $\mathrm{Fe}$ (a)

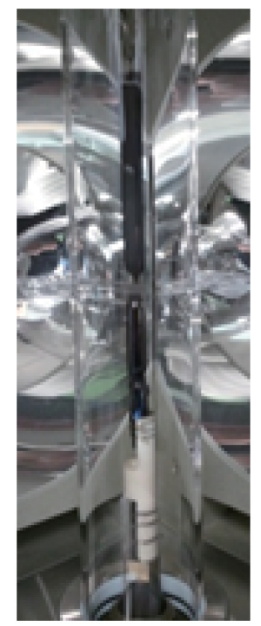

(b)

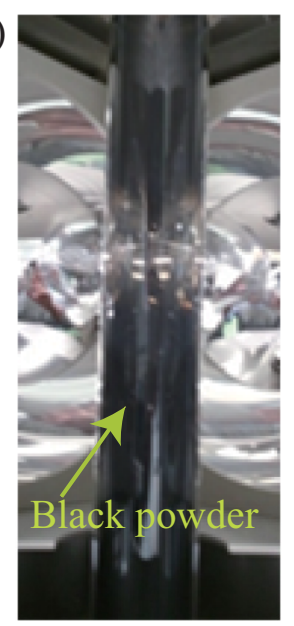

FIG. 6. The FZ equipment (a) before and (b) after crystal growth. The black powder attached on the surface of the quartz tube after the crystal growth.
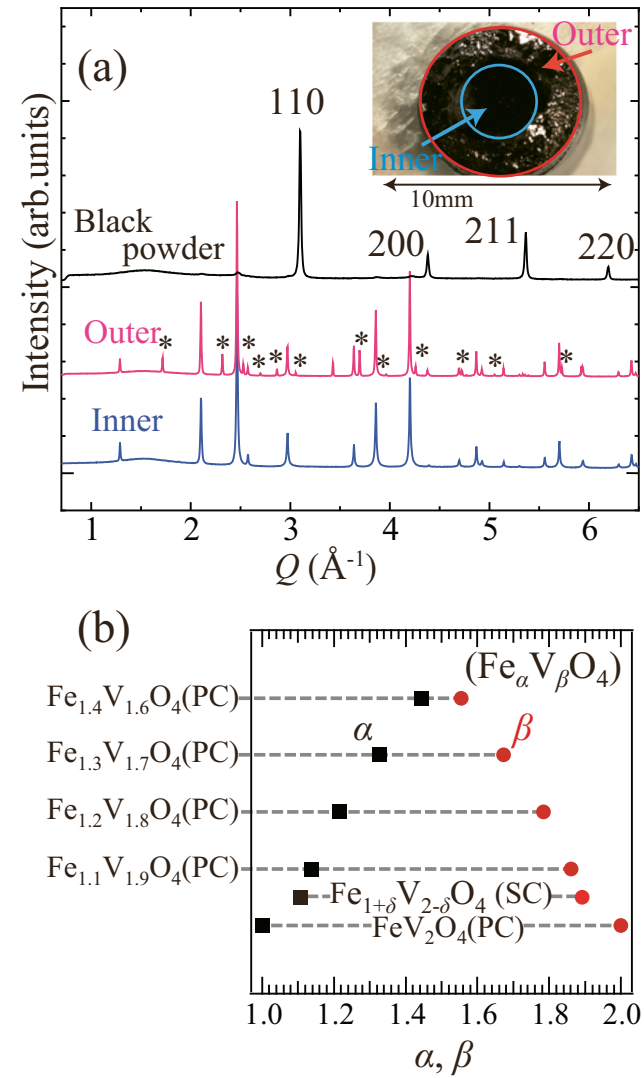

FIG. 7. (a) SPD patterns for the black powder and the outer and inner regions of the crystal. The Bragg peaks of black powder can be indexed as a cubic system with the space group $\operatorname{Im} \overline{3} m$. The asterisks indicate the Bragg peaks derived from the impurity of $\mathrm{V}_{2} \mathrm{O}_{3}$. The inset shows the section of seed rods after crystal growth. (b) The ratio of $\mathrm{Fe}(\alpha)$ and $\mathrm{V}(\beta)$ cations was determined by the chemical composition analysis. Note that PC and SC denote the polycrystalline and the single-crystal samples, respectively.

$(5 \%-10 \%)$ even in the inner region of the growth crystal. This excess $\mathrm{Fe}$ in the single crystal may originate from the phase separation of $\mathrm{V}_{2} \mathrm{O}_{3}$ in the outer region of the crystal, as shown in Fig. 7(a). As a result, the off-stoichiometry in the single crystal is derived from both the evaporation of Fe and the precipitation of $\mathrm{V}_{2} \mathrm{O}_{3}$ during the crystal growth.

The chemical compositions of the single-crystal and polycrystalline samples were determined by energy dispersion $\mathrm{X}$-ray spectroscopy by using Horiba EMAX ENERGY equipment. Figure 7(b) shows the ratio of $\mathrm{Fe}(\alpha)$ and $\mathrm{V}(\beta)$ cations, where $\alpha+\beta=3$, in the single crystal in inner region of growth crystal and the polycrystalline sample of $\mathrm{FeV}_{2} \mathrm{O}_{4}$ and of $(\mathrm{Fe})\left[\mathrm{V}_{2-x} \mathrm{Fe}_{x}\right] \mathrm{O}_{4}$. The $\alpha$ values for the polycrystalline and single-crystal samples of $\mathrm{FeV}_{2} \mathrm{O}_{4}$ are 1.001 (8) and 1.100 (9), respectively. These results indicate a stoichiometric mixture for the polycrystalline sample of $\mathrm{FeV}_{2} \mathrm{O}_{4}$ and the existence of excess $\mathrm{Fe}$ in the single-crystal sample. The chemical composition of the single-crystal sample is similar to that of the polycrystalline sample of $\mathrm{Fe}\left[\mathrm{V}_{1.9} \mathrm{Fe}_{0.1}\right] \mathrm{O}_{4}$. This result is consistent with the results of the measurements of physical property. Hence, we regard the single crystal of $\mathrm{FeV}_{2} \mathrm{O}_{4}$ as $\mathrm{Fe}_{1+\delta} \mathrm{V}_{2-\delta} \mathrm{O}_{4}$, and the $\delta$ is about 0.1 . Note that we focused on 
the ratio of the transition metals in this study because it was difficult to quantitatively evaluate the oxygen atom.

\section{APPENDIX B: PHYSICAL PROPERTIES IN $\mathrm{Fe}_{1+\delta} \mathrm{V}_{2-\delta} \mathrm{O}_{4}$ SINGLE CRYSTAL}

We have investigated the structural properties in the $\mathrm{Fe}_{1+\delta} \mathrm{V}_{2-\delta} \mathrm{O}_{4}$ single crystal at low temperatures using the synchrotron diffraction technique. Figure 8 represents the synchrotron diffraction patterns around the 800 cubic Bragg peak. At $160 \mathrm{~K}$, the 800 reflection is a single peak, and the peak is split into two peaks at $110 \mathrm{~K}$ and three peaks at $100 \mathrm{~K}$. Furthermore, the three peaks are merged into two peaks at $60 \mathrm{~K}$. For the $\mathrm{Fe}_{1+\delta} \mathrm{V}_{2-\delta} \mathrm{O}_{4}$ single crystal, the temperature dependencies of the lattice constants, which are estimated from the Bragg peak positions, magnetization, and specific heat are shown in Figs. 8(b) and 8(c). Note that the experimental data of the temperature dependencies of the magnetization and the specific heat are from Ref. [17]. These results agree perfectly with recent studies using the single crystal [11,13]. On the other hand, below $40 \mathrm{~K}$, we could not clearly observe a peak splitting caused by orthorhombic distortion. The reason is that the excess Fe in the single crystal suppresses the orthorhombic distortion at the lowest temperature. Indeed, the structural properties and phase transition temperatures for the single crystal are very similar to those for $\mathrm{Fe}\left[\mathrm{V}_{1.9} \mathrm{Fe}_{0.1}\right] \mathrm{O}_{4}$.

The excess $\mathrm{Fe}$ in the single crystal can affect the transitions at $T_{\mathrm{N} 1}$ and $T_{\mathrm{N} 2}$. In practice, in the specific heat, the peak at $T_{\mathrm{N} 2}$ in the polycrystalline sample is much sharper and shifts to higher temperature than that in the single crystal, as shown in Fig. 8(d). Additionally, the lattice constants around $T_{\mathrm{N} 2}$ in the polycrystalline sample exhibit a sharper drop with decreasing temperature than those in the single crystal, as shown in Fig. 8(e). These differences of the behaviors between the polycrystalline sample and the single crystal can be explained by the excess $\mathrm{Fe}$ with nonorbital degrees of freedom at the $B$ site in the spinel structure in the single crystal [34]. The phase transition at $T_{\mathrm{N} 2}$ originates from the magnetic and orbital orders of $\mathrm{V}^{3+}$ ions at the $B$ site, which play an important role in this transition; therefore, the excess $\mathrm{Fe}$ in the single crystal suppresses the long-range magnetic and the orbital orders at $T_{\mathrm{N} 2}$, leading to the difference in the behaviors of the specific heat between the polycrystalline and single-crystal samples. On the other hand, the peak of the specific heat at $T_{\mathrm{N} 1}$ in the single crystal is slightly sharper than that in the polycrystalline sample. At present, the origin of this behavior is unclear. However, the transition temperature slightly increases. The increase in the transition temperature was also observed in a Fe-rich system, $\mathrm{Fe}_{1+x} \mathrm{~V}_{2-x} \mathrm{O}_{4}$. In this
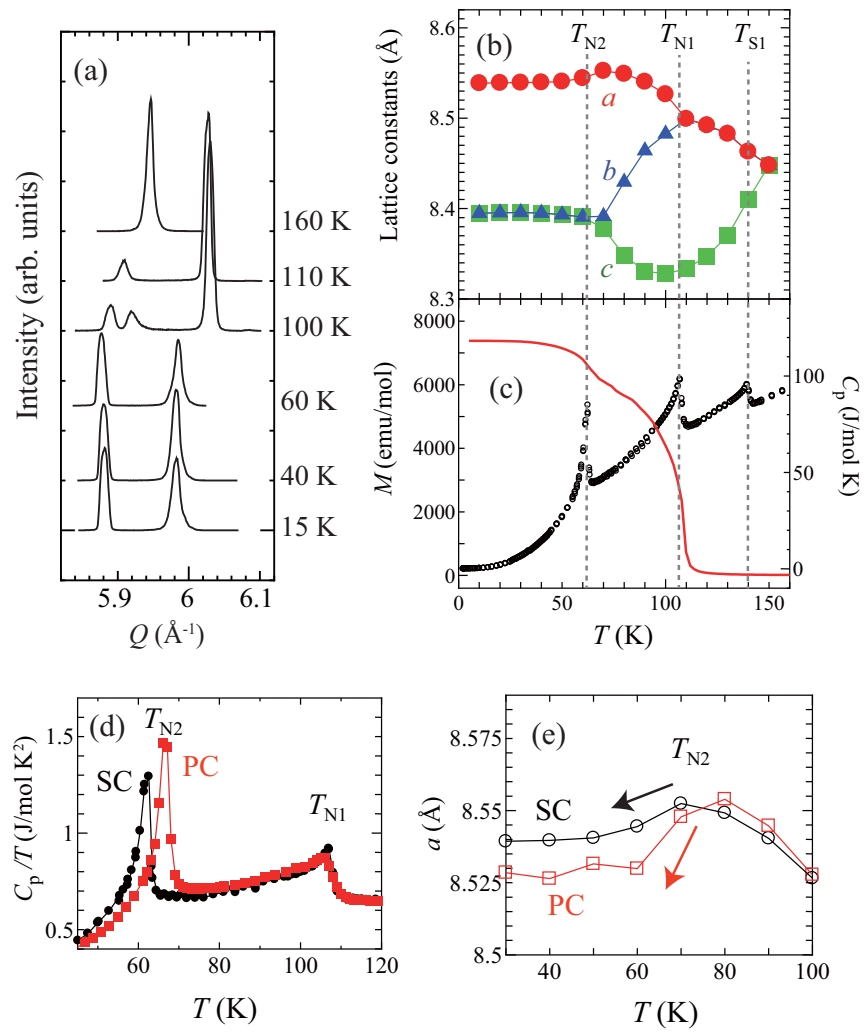

FIG. 8. (a) Single-crystalline synchrotron diffraction patterns around the 800 cubic Bragg peak. (b) Temperature dependencies of lattice constants. (c) Temperature dependencies of the magnetization and the specific heat for $\mathrm{Fe}_{1+\delta} \mathrm{V}_{2-\delta} \mathrm{O}_{4}$ single crystal [17]. Temperature dependencies of (d) the specific heat and (e) the lattice constant of the $a$ axis for single-crystal (SC) and polycrystalline (PC) $\mathrm{FeV}_{2} \mathrm{O}_{4}$ samples.

viewpoint, the magnetic interaction between the $A$ site and the $B$ site in the spinel structure may be enhanced by the excess Fe with $S=5 / 2$ [34]. In $\mathrm{FeV}_{2} \mathrm{O}_{4}$, the magnetic states are strongly related to the orbital states at $T_{\mathrm{N} 1}$; therefore, the excess $\mathrm{Fe}$ in the single crystal affects the transitions related with orbital and spin degrees of freedom. Note that the change of temperature dependence of the magnetization at $T_{\mathrm{N} 1}$ and $T_{\mathrm{N} 2}$ in the $\mathrm{Fe}_{1+\delta} \mathrm{V}_{2-\delta} \mathrm{O}_{4}$ single crystal is much sharper than that in the polycrystalline sample. This behavior probably comes from the strong magnetic anisotropy in $\mathrm{FeV}_{2} \mathrm{O}_{4}$ [17]. In this study, the magnetic field was applied along the $[001]_{c}$ direction of the crystal, where this direction is along the easy axis of the magnetization in $\mathrm{FeV}_{2} \mathrm{O}_{4}[14,17]$.
[1] P. G. Radaelli, D. E. Cox, M. Marezio, and S. W. Cheong, Phys. Rev. B 55, 3015 (1997).

[2] Y. Tokura and N. Nagaosa, Science 288, 462 (2000).

[3] S. Niitaka, H. Ohsumi, K. Sugimoto, S. Lee, Y. Oshima, K. Kato, D. Hashizume, T. Arima, M. Takata, and H. Takagi, Phys. Rev. Lett. 111, 267201 (2013).

[4] H. Tsunetsugu and Y. Motome, Phys. Rev. B 68, 060405 (2003).
[5] O. Tchernyshyov, Phys. Rev. Lett. 93, 157206 (2004).

[6] T. Maitra and R. Valenti, Phys. Rev. Lett. 99, 126401 (2007).

[7] T. Suzuki, M. Katsumura, K. Taniguchi, T. Arima, and T. Katsufuji, Phys. Rev. Lett. 98, 127203 (2007).

[8] V. O. Garlea, R. Jin, D. Mandrus, B. Roessli, Q. Huang, M. Miller, A. J. Schultz, and S. E. Nagler, Phys. Rev. Lett. 100, 066404 (2008). 
[9] S. Sarkar, T. Maitra, R. Valenti, and T. Saha-Dasgupta, Phys. Rev. Lett. 102, 216405 (2009).

[10] G.-W. Chern, N. Perkins, and Z. Hao, Phys. Rev. B 81, 125127 (2010).

[11] Y. Nii, H. Sagayama, T. Arima, S. Aoyagi, R. Sakai, S. Maki, E. Nishibori, H. Sawa, K. Sugimoto, H. Ohsumi, and M. Takata, Phys. Rev. B 86, 125142 (2012).

[12] T. Katsufuji, T. Suzuki, H. Takei, M. Shingu, K. Kato, K. Osaka, M. Takata, H. Sagayama, and T. Arima, J. Phys. Soc. Jpn. 77, 053708 (2008).

[13] G. J. MacDougall, V. O. Garlea, A. A. Aczel, H. D. Zhou, and S. E. Nagler, Phys. Rev. B 86, 060414 (2012).

[14] H. Takei, T. Suzuki, and T. Katsufuji, Appl. Phys. Lett. 91, 072506 (2007).

[15] A. Kismarahardja, J. S. Brooks, H. D. Zhou, E. S. Choi, K. Matsubayashi, and Y. Uwatoko, Phys. Rev. B 87, 054432 (2013).

[16] Q. Zhang, K. Singh, F. Guillou, C. Simon, Y. Breard, V. Caignaert, and V. Hardy, Phys. Rev. B 85, 054405 (2012).

[17] S. Kawaguchi, H. Ishibashi, S. Nishihara, M. Miyagawa, K. Inoue, S. Mori, and Y. Kubota, J. Phys.: Condens. Matter 25, 416005 (2013).

[18] J.-S. Kang, J. Hwang, D. H. Kim, E. Lee, W. C. Kim, C. S. Kim, S. Kwon, S. Lee, J.-Y. Kim, T. Ueno, M. Sawada, B. Kim, B. H. Kim, and B. I. Min, Phys. Rev. B 85, 165136 (2012).

[19] S. Sarkar and T. Saha-Dasgupta, Phys. Rev. B 84, 235112 (2011).

[20] G. J. MacDougall, I. Brodsky, A. A. Aczel, V. O. Garlea, G. E. Granroth, A. D. Christianson, T. Hong, H. D. Zhou, and S. E. Nagler, Phys. Rev. B 89, 224404 (2014).

[21] Q. Zhang, M. Ramazanoglu, S. Chi, Y. Liu, T. A. Lograsso, and D. Vaknin, Phys. Rev. B 89, 224416 (2014).

[22] E. Nishibori et al., Nucl. Instrum. Methods Phys. Res., Sect. A 467-468, 1045 (2001).
[23] M. R. Suchomel, D. P. Shoemaker, L. Ribaud, M. C. Kemei, and R. Seshadri, Phys. Rev. B 86, 054406 (2012).

[24] J. H. Van Vleck, J. Chem. Phys. 7, 72 (1939).

[25] U. Öpik and M. H. L. Pryce, Proc. R. Soc. London, Ser. A 238, 425 (1957).

[26] J. B. Goodenough, J. Phys. Chem. Solids 25, 151 (1964).

[27] S. Ohtani, Y. Watanabe, M. Saito, N. Abe, K. Taniguchi, H. Sagayama, T. Arima, M. Watanabe, and Y. Noda, J. Phys.: Condens. Matter 22, 176003 (2010).

[28] M. Kataoka and J. Kanamori, J. Phys. Soc. Jpn. 32, 113 (1972).

[29] S. Kawaguchi, H. Ishibashi, and Y. Kubota, J. Phys. Soc. Jpn. 83, 074601 (2014).

[30] S. Kawaguchi, H. Ishibashi, F. Miki, and Y. Kubota, JPS Conf. Proc. 1, 012084 (2014).

[31] S. Kawaguchi, H. Ishibashi, J. Kim, and Y. Kubota, J. Phys.: Condens. Matter 26, 346001 (2014).

[32] D. Choudhury, T. Suzuki, D. Okuyama, D. Morikawa, K. Kato, M. Takata, K. Kobayashi, R. Kumai, H. Nakao, Y. Murakami, M. Bremholm, B. B. Iversen, T. Arima, Y. Tokura, and Y. Taguchi, Phys. Rev. B 89, 104427 (2014).

[33] N. Liu, K. H. Zhao, X. L. Shi, and L. W. Zhang, J. Appl. Phys. 111, 124112 (2012).

[34] M. Abe, M. Kawachi, and S. Nomura, J. Solid State Chem. 10, 351 (1974).

[35] A. Kiswandhi, J. S. Brooks, J. Lu, J. Whalen, T. Siegrist, and H. D. Zhou, Phys. Rev. B 84, 205138 (2011).

[36] A. Kismarahardja, J. S. Brooks, A. Kiswandhi, K. Matsubayashi, R. Yamanaka, Y. Uwatoko, J. Whalen, T. Siegrist, and H. D. Zhou, Phys. Rev. Lett. 106, 056602 (2011).

[37] D. B. Rogers, R. J. Arnott, A. Wold, and J. B. Goodenough, J. Phys. Chem. Solids 24, 347 (1963). 